\title{
Predictors of mortality in solid-organ transplant recipients with infections caused by Acinetobacter baumannii
}

This article was published in the following Dove Press journal:

Therapeutics and Clinical Risk Management

2I August 2015

Number of times this article has been viewed

Hua Liu'

Qifa $\mathrm{Ye}^{2,3}$

Qiquan Wan ${ }^{2}$

Jiandang Zhou ${ }^{4}$

'Central Sterile Supply Department, ${ }^{2}$ Department of Transplant Surgery, Third Xiangya Hospital, Central South University, Changsha, ${ }^{3}$ Department of Transplant Surgery, Zhongnan Hospital, Wuhan University, Wuhan, ${ }^{4}$ Department of Clinical Laboratory of Microbiology, Third Xiangya Hospital, Central South University, Changsha, People's Republic of China
Correspondence: Qiquan Wan Department of Transplant Surgery, Third Xiangya Hospital, Central South University, 138 Tongzipo Road, Changsha, Hunan 4I0013, People's Republic of China

Tel +8673 I 886I 83I2

Fax +8673188618312

Email I3548685542@I63.com
Abstract: Acinetobacter baumannii can cause a serious infection in solid-organ transplant (SOT) recipients, and more data on $A$. baumannii infection is needed. We sought to investigate the epidemiology and distribution of $A$. baumannii isolates in SOT recipients. We also investigated the risk factors for overall in-hospital mortality and infection-related 30-day mortality using multivariate logistic regression analysis. A double-center retrospective study of SOT recipients who were infected with A. baumannii between January 2003 and January 2015 was conducted. A total of 71 individuals developed 93 episodes of $A$. baumannii infection, with a mean age of 44.5 years ( $44.5 \pm 11.9$ years). Ninety percent of recipients had nosocomial origin A. baumannii infection, with the bloodstream as the most common site of infection (32.4\%). Septic shock developed in $23.9 \%$ (17 of 71 ) of all recipients with $A$. baumannii infection. Morbidity and mortality rates of $A$. baumannii infections were high in SOT recipients. The incidence rate of A. baumannii infection in SOT recipients was 3.9\% (71 of 1,821). Overall in-hospital mortality and infection-related 30-day mortality were 53.5\% (38 of 71) and 40.8\% (29 of 71), respectively. Risk factors independently associated with overall in-hospital mortality were mechanical ventilation at onset of $A$. baumannii infection (odds ratio [OR] 6.29, 95\% confidence interval [CI] 1.48-26.85; $P=0.013$ ), liver or liver-kidney transplantation (OR 15.33, 95\% CI 1.82-129.18; $P=0.012$ ), and late-onset $A$. baumannii infection (OR 7.61, 95\% CI 1.07-54.36; $P=0.043$ ). A platelet count $<50,000 / \mathrm{mm}^{3}(\mathrm{OR} 12.76,95 \%$ CI $1.28-126.81 ; P=0.030)$ and mechanical ventilation at onset of $A$. baumannii infection (OR 189.98, 95\% CI 13.23-2,728.81; $P<0.001$ ) were identified as independent risk factors for infection-related 30-day mortality. In conclusion, the morbidity and mortality rates of $A$. baumannii infections were high in SOT recipients. Mechanical ventilation at onset of $A$. baumannii infection was associated with higher overall in-hospital mortality and infection-related mortality. For overall in-hospital mortality, liver or liver-kidney transplantation and late-onset $A$. baumannii infection, and for infection-related mortality, thrombocytopenia were also risk factors, respectively.

Keywords: A. baumannii, infection, predictors, mortality, solid-organ transplantation

\section{Background}

Bacteria can cause serious infection after solid-organ transplantation (SOT), with significant morbidity and mortality rates. ${ }^{1-4}$ The overall infection rate of Acinetobacter baumannii infection among SOT recipients is $1.4 \%-6.1 \%$, and the mortality rate ranges from $39 \%$ to $80 \% .^{5-10}$

A. baumannii is a non-lactose-fermenting Gram-negative bacilli widely distributed in nature. It is an increasingly resilient opportunistic pathogen in critically ill or immunocompromised patients, associated with soft-tissue infection, catheter-associated bacteremia, ventilator-associated pneumonia, urinary tract infection, and peritonitis. ${ }^{1-13}$ 
A. baumannii is particularly problematic, due to its capacity of persisting in the hospital environment, including among health care workers, resulting in clonal outbreaks. ${ }^{14-17}$

Although SOT recipients are particularly susceptible to A. baumannii infection with high morbidity and mortality, little has been published regarding the impact and features of A. baumannii infections..$^{5,6,8,12,18}$ Herein, in the largest samplesize cohort study specifically focusing on $A$. baumannii infections in SOT recipients so far, our objective was to determine the epidemiology and risk factors for mortality.

\section{Materials and methods}

\section{Ethics statement}

The study protocol was approved by the Third Xiangya Hospital, Central South University and the Zhongnan Hospital, Wuhan University Medical Ethical Committee prior to patient identification and data collection.

\section{Study population}

This study was conducted at both the Third Xiangya Hospital, an 1,800-bed teaching hospital, affiliated with Central South University, Changsha, People's Republic of China (PRC), with an active abdominal SOT program (annual average of 30 liver and 150 kidney transplants) and Zhongnan Hospital, a 1,200-bed teaching hospital, affiliated with Wuhan University, Wuhan, PRC, with an active abdominal SOT program (annual average of 40 liver and 100 kidney transplants). The medical records of SOT recipients with symptomatic A. baumannii infection occurring between January 1, 2003 and January 1, 2015 at both hospitals were retrospectively reviewed. Maintenance immunosuppression was based on calcineurin inhibitors (cyclosporine or tacrolimus) and corticosteroids, with or without mycophenolate mofetil/ azathioprine.

\section{Study design and data collection}

We performed a retrospective study to investigate the epidemiology of pathogens and risk factors for overall in-hospital mortality and infection-related 30-day mortality in SOT recipients with $A$. baumannii infection. Demographic and clinical characteristics included age, sex, induction immunosuppressives, body temperature at the onset of infection, site and date of diagnosis of $A$. baumannii infection, nosocomial origin of infection, empirical antimicrobial therapy, acute rejection, reoperation, multidrug-resistant (MDR) A. baumannii, intensive care unit (ICU) stay, mechanical ventilation, and septic shock, and the laboratory records of these recipients were analyzed. The laboratory variables were collected within the first 24 hours after the culture was drawn, including serum creatinine and albumin levels and white blood cell, platelet, and lymphocyte counts. All episodes of $A$. baumannii infection were reviewed, and only the first episode was included for further statistical analyses. The follow-up time of all recipients was at least 1 month after the onset of $A$. baumannii infection.

\section{Definitions}

The presence of infection, including bacteremia, pneumonia, peritonitis, pleuritis, vascular catheter and urinary tract infection, was defined based on the criteria suggested by the US Centers for Disease Control and Prevention. ${ }^{19}$ Appropriate antimicrobial therapy was defined as use of a drug to which the isolated pathogen was susceptible in vitro within 48 hours of sampling for culture. ${ }^{20}$ Infection was considered to be nosocomially acquired in patients who had been hospitalized for 48 hours or longer. Infection was categorized as early onset if it occurred 2 months (60 days) or less after SOT, or late onset if it occurred $>2$ months after SOT. ${ }^{21}$ MDR $A$. baumannii was defined as acquired nonsusceptibility to at least one agent in three or more antimicrobial categories, which included aminoglycosides, carbapenems, extended-spectrum cephalosporins, fluoroquinolones, antipseudomonal penicillins + $\beta$-lactamase inhibitors, ampicillin-sulbactam, trimethoprimsulfamethoxazole, tetracyclines, and polymyxins. ${ }^{22}$ Septic shock was diagnosed in recipients who had a positive culture and developed persistent dysfunction of at least one organ caused by hypoperfusion that was unresponsive to intravenous fluid challenge and unexplained by other causes. ${ }^{23}$ Mortality was regarded as related to infection when death was associated with clinical signs of active $A$. baumannii infection without evidence of any other cause. ${ }^{24}$

\section{Statistical analysis}

Continuous variables are expressed as medians \pm standard deviation. The $\chi^{2}$ and Fisher exact tests were used to analyze categorical variables. Overall in-hospital mortality rates and infection-related 30-day mortality rates were calculated, respectively. Univariate analysis was applied to examine the association between demographic/clinical variables and overall in-hospital/infection-related mortality. We included variables identified $(P<0.10)$ by univariate analyses. Odds ratios (ORs) with 95\% confidence interval (CIs) were calculated. Statistical analyses were performed using SPSS for Windows, version 22.0 (IBM Corporation, Armonk, NY, USA). Statistical significance was defined as $P<0.05$ (two-tailed). 


\section{Results}

During the 12-year study period, 1,502 SOT recipients 1,305 kidney, 189 liver, three heart, four simultaneous liver-kidney, and one simultaneous kidney-pancreas recipients - in the Third Xiangya Hospital and 319 SOT recipients - 239 kidney, 78 liver, and two simultaneous liver-kidney recipients - in Zhongnan Hospital were enrolled.

A total of 93 episodes of $A$. baumannii infection occurred in 71 SOT recipients: 48 were kidney-transplant recipients (67.6\%), 21 liver recipients (29.6\%), and two simultaneous liver-kidney recipients $(2.8 \%)$ (Table 1$)$. The incidence

Table I Demographic, laboratory, and clinical variables of 7 I SOT patients with Acinetobacter baumannii infection

\begin{tabular}{|c|c|}
\hline Characteristics & Value \\
\hline Age, mean years $\pm S D$ & $44.5 \pm 11.9$ \\
\hline Male sex, n (\%) & $53(74.6)$ \\
\hline Temperature $39^{\circ} \mathrm{C}$ or greater, $\mathrm{n}(\%)$ & $17(23.9)$ \\
\hline Nosocomial origin, $\mathrm{n}(\%)$ & $64(90.1)$ \\
\hline Inappropriate antimicrobial use, $\mathrm{n}(\%)$ & $4 \mathrm{I}(57.7)$ \\
\hline Induction therapy, n (\%) & $19(26.8)$ \\
\hline Acute rejection prior to infection, $\mathrm{n}(\%)$ & $13(18.3)$ \\
\hline High-dose steroids for rejection before infection, $\mathrm{n}(\%)$ & $8(11.3)$ \\
\hline Reoperation, n (\%) & $7(9.9)$ \\
\hline Carbapenem-resistant A. baumannii, n (\%) & $40(56.3)$ \\
\hline MDR A. baumannii, n (\%) & $53(74.6)$ \\
\hline ICU stay, n (\%) & $51(7 \mid .8)$ \\
\hline Mechanical ventilation, $\mathrm{n}(\%)$ & $33(46.5)$ \\
\hline Septic shock, n (\%) & $17(23.9)$ \\
\hline \multicolumn{2}{|l|}{ Type of transplantation, $\mathrm{n}(\%)$} \\
\hline Kidney & $48(67.6)$ \\
\hline Liver & $21(29.6)$ \\
\hline Liver-kidney & $2(2.8)$ \\
\hline \multicolumn{2}{|l|}{ Site of infection, $\mathrm{n}(\%)$} \\
\hline Bloodstream & $23(32.4)$ \\
\hline Lung & $20(28.2)$ \\
\hline Intra-abdomen/biliary tract & $8(11.3)$ \\
\hline Urinary tract & $3(4.2)$ \\
\hline Vascular catheter & $\mathrm{I}(\mathrm{l} .4)$ \\
\hline Multiple culture-positive sites & $16(22.5)$ \\
\hline \multicolumn{2}{|l|}{ Patient immunosuppressant treatment, $\mathrm{n}(\%)$} \\
\hline Tacrolimus & $62(87.3)$ \\
\hline Cyclosporine A & $8(11.3)$ \\
\hline \multicolumn{2}{|l|}{ Time of infection onset, $\mathrm{n}(\%)$} \\
\hline$<2$ months posttransplant (early onset) & $4 \mid(57.7)$ \\
\hline$\geq 2$ months posttransplant (late onset) & $30(42.3)$ \\
\hline \multicolumn{2}{|l|}{ Laboratory variables from blood, n (\%) } \\
\hline Platelet count $<50,000 / \mathrm{mm}^{3}$ & $24(33.8)$ \\
\hline Lymphocyte count $<300 / \mathrm{mm}^{3}$ & $23(32.4)$ \\
\hline Albumin $<30 \mathrm{~g} / \mathrm{L}$ & $12(16.9)$ \\
\hline WBC count $>15,000 / \mathrm{mm}^{3}$ & $22(31.0)$ \\
\hline Creatinine $>1.5 \mathrm{mg} / \mathrm{dL}$ & $4 \mathrm{I}(57.7)$ \\
\hline Related mortality, n (\%) & $29(40.8)$ \\
\hline Overall in-hospital mortality, n (\%) & $38(53.5)$ \\
\hline
\end{tabular}

Abbreviations: SOT, solid-organ transplantation; SD, standard deviation MDR, multidrug-resistant; ICU, intensive care unit; WBC, white blood cell. rate of A. baumannii infection in SOT recipients was 3.9\% (71 of 1,821 ), with the rate of $3.1 \%$ in kidney recipients and $8.4 \%$ in liver or simultaneous liver-kidney recipients. High-dose steroids ( $\geq 750 \mathrm{mg}$ ) were used in eight (11.3\%) recipients for acute rejection before $A$. baumannii infection . Tacrolimus and cyclosporine A were used in 62 (87.3\%) and eight $(11.3 \%)$ recipients, respectively. The remaining patient did not use any calcineurin inhibitors between liver transplantation and the onset of $A$. baumannii infection, due to a severe clinical condition. Among these 93 episodes of A. baumannii infection, the presumed sources of infection were the bloodstream ( $n=35,37.6 \%)$, lung $(n=33,35.5 \%)$, urinary tract $(\mathrm{n}=16,17.2 \%)$, intra-abdomen/biliary tract $(\mathrm{n}=6$, $6.5 \%)$, thoracic cavity $(n=2,2.2 \%)$, and catheter $(n=1,1.1 \%)$. The median time to onset of $A$. baumannii infection after SOT transplantation was 38 days (interquartile range 8-111 days), and $61.3 \%$ (57 of 93) occurred as early onset.

The mean age for SOT recipients with $A$. baumannii

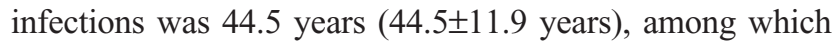
$53(74.6 \%)$ were male. Ninety percent of recipients had nosocomial origin A. baumannii infection. Seventeen patients (23.9\%) had a body temperature of $39^{\circ} \mathrm{C}$ or higher at the onset of $A$. baumannii infection. The most common cases were recipients with $A$. baumannii bacteremias (23 cases, 32.4\%), pneumonia (20 cases, $28.2 \%$ ), and multiple culture-positive site infections ( 16 cases, $22.5 \%$ ). Seventy-six percent ( 71 of 93) of $A$. baumannii were carbapenem-resistant. As far as the number of infected patients with carbapenem-resistant strains was concerned, $40(56.3 \%)$ had carbapenem-resistant strains involved. Fifty-three recipients (74.6\%) developed MDR $A$. baumannii infection. Forty-one patients (57.7\%) received inappropriate antibiotic treatment. Forty-one patients $(57.7 \%)$ had $A$. baumannii infection within the first 2 months after SOT. At the onset of A. baumannii infection, $71.8 \%$ and $46.5 \%$ of cases needed ICU stay and mechanical ventilation support, respectively. SOT recipients with late-onset $A$. baumannii infection needed more frequent ICU stays (24 of 30 [80.0\%] vs 27 of 41 [65.8\%]) and mechanical ventilation support (21 of 30 [70.0\%] vs 10 of 41 [24.4\%]) than those with early onset infection. Septic shock developed in $23.9 \%$ (17 of 71) of all recipients with $A$. baumannii infection and in $37.1 \%$ (13 of 35) of all recipients with $A$. baumannii bacteremias. Infection was associated with high overall in-hospital mortality (53.5\%) and infection-related 30-day mortality (40.8\%). Forty-one, 22, 24, and 23 recipients had a serum creatinine level $>1.5 \mathrm{mg} / \mathrm{dL}$, white blood cell count $>15,000 / \mathrm{mm}^{3}$, platelet count $<50,000 / \mathrm{mm}^{3}$, and lymphocyte count $<300 / \mathrm{mm}^{3}$, respectively. The basic demographic, laboratory, and clinical 
Table 2 Risk factors for overall in-hospital mortality in SOT recipients with Acinetobacter baumannii infection

\begin{tabular}{|c|c|c|c|c|}
\hline Characteristics & Overall mortality & Survival & $P$-value & OR $(95 \% \mathrm{Cl})$ \\
\hline Total, n (\%) & $38(53.5)$ & $33(46.5)$ & & \\
\hline \multicolumn{5}{|l|}{ Univariate analysis, n (\%) } \\
\hline Age $\geq 40$ years & $27(7 I . I)$ & $22(66.7)$ & 0.690 & $1.23(0.45-3.36)$ \\
\hline Male sex & $26(68.4)$ & $27(81.8)$ & 0.200 & $0.48(0.16-1.47)$ \\
\hline Temperature $\geq 39^{\circ} \mathrm{C}$ & $12(31.6)$ & $5(15.2)$ & 0.112 & $2.59(0.80-8.34)$ \\
\hline Inappropriate antibiotics & $19(50.0)$ & $22(66.7)$ & 0.159 & $0.50(0.19-1.31)$ \\
\hline Nosocomial infection & $35(92.1)$ & $29(87.9)$ & 0.554 & $1.61(0.33-7.78)$ \\
\hline Liver or liver-kidney transplant & $17(44.7)$ & $6(18.2)$ & 0.020 & $3.64(1.22-10.85)$ \\
\hline Multiple culture-positive sites & II (28.9) & $5(15.2)$ & 0.171 & $2.28(0.70-7.44)$ \\
\hline Late-onset infection & $20(52.6)$ & $10(30.3)$ & 0.060 & $2.56(0.96-6.80)$ \\
\hline Acute rejection & $6(15.8)$ & $7(2 \mid .2)$ & 0.557 & $0.70(0.2 \mathrm{I}-2.33)$ \\
\hline Induction therapy & $8(21.1)$ & II (33.3) & 0.531 & $0.71(0.25-2.05)$ \\
\hline Reoperation & $5(13.2)$ & $2(6.1)$ & 0.328 & $2.35(0.42-13.01)$ \\
\hline MDR A. baumannii & $29(76.3)$ & $24(72.7)$ & 0.729 & $1.21(0.4 \mid-3.53)$ \\
\hline ICU stay & $35(92.1)$ & $16(48.5)$ & $<0.001$ & $12.40(3.17-48.42)$ \\
\hline Mechanical ventilation & $27(7 I . I)$ & $6(18.2)$ & $<0.001$ & II.04 (3.57-34.15) \\
\hline Septic shock & $15(39.5)$ & $2(6.1)$ & 0.004 & $10.11(2.10-48.63)$ \\
\hline Platelet count $<50,000 / \mathrm{mm}^{3}$ & $20(52.6)$ & $4(12.1)$ & 0.001 & $8.06(2.37-27.40)$ \\
\hline Lymphocyte count $<300 / \mathrm{mm}^{3}$ & $16(42.1)$ & $7(21.2)$ & 0.065 & $2.70(0.94-7.75)$ \\
\hline Albumin $<30 \mathrm{~g} / \mathrm{L}$ & $10(26.3)$ & $2(6.1)$ & 0.036 & $5.54(1.12-27.47)$ \\
\hline WBC count $\geq 15,000 / \mathrm{mm}^{3}$ & $10(26.3)$ & $12(36.4)$ & 0.690 & $0.82(0.30-2.23)$ \\
\hline Creatinine $\geq 1.5 \mathrm{mg} / \mathrm{dL}$ & $26(68.4)$ & $15(45.5)$ & 0.053 & $2.60(0.99-6.85)$ \\
\hline \multicolumn{5}{|l|}{ Multivariate analysis } \\
\hline Liver or liver-kidney transplant & & & 0.012 & $15.33(1.82-129.18)$ \\
\hline Late-onset infection & & & 0.043 & $7.61(1.07-54.36)$ \\
\hline Mechanical ventilation & & & 0.013 & $6.29(1.48-26.85)$ \\
\hline
\end{tabular}

Abbreviations: SOT, solid-organ transplantation; OR, odds ratio; $\mathrm{Cl}$, confidence interval; MDR, multidrug-resistant; ICU, intensive care unit; WBC, white blood cell.

characteristics of all SOT recipients with A. baumannii infection are shown in Table 1.

Tables 2 and 3 present the risk factors for overall in-hospital and infection-related mortality, respectively. In univariate analysis, liver or simultaneous liver-kidney transplantation $(P=0.02)$, ICU stay $(P<0.001)$, mechanical ventilation $(P<0.001)$, septic shock $(P=0.004)$, albumin level $<30 \mathrm{~g} / \mathrm{L}$ ( $P=0.036)$, and platelet count $<50,000 / \mathrm{mm}^{3}(P=0.001)$ were more likely to develop overall in-hospital mortality. In multivariate logistic regression analysis of these data, mechanical ventilation at onset of $A$. baumannii infection (OR 6.29, 95\% CI 1.48-26.85; $P=0.013$ ), liver or simultaneous liver-kidney transplantation (OR 15.33, 95\% CI 1.82-129.18; $P=0.012$ ), and late-onset $A$. baumannii infection (OR 7.61, 95\% CI 1.07-54.36; $P=0.043$ ) were all associated with a greater risk of overall in-hospital mortality. In univariate analysis, the following variables were significantly associated with infection-related 30-day mortality: temperature $\geq 39^{\circ} \mathrm{C}$ ( $P=0.026)$, late-onset $A$. baumannii infection $(P=0.022)$, ICU stay $(P=0.003)$, mechanical ventilation $(P<0.001)$, septic shock $(P<0.001)$, creatinine level $>1.5 \mathrm{mg} / \mathrm{dL}(P=0.041)$, and platelet count $<50,000 / \mathrm{mm}^{3}(P=0.002)$. In multivariate analysis, platelet count $<50,000 / \mathrm{mm}^{3}$ (OR $12.76,95 \%$ CI $1.28-126.81 ; P=0.030)$ and mechanical ventilation at onset of A. baumannii infection (OR 189.98, 95\% CI 13.23-2,728.81; $P<0.001)$ were significantly related to a higher risk of 30-day mortality associated with $A$. baumannii infection.

\section{Discussion}

Invasive bacterial infections have become a leading contributor to patient morbidity and mortality among SOT recipients. ${ }^{25} \mathrm{~A}$. baumannii is an increasing problem among SOT recipients. Not only is A. baumannii environmentally resilient but this pathogen can also readily develop antibiotic resistance. van Delden et al reported that the prevalence of MDR Acinetobacter is increasing, and is related to higher rates of treatment failure in SOT recipients. ${ }^{26}$

The incidence rate of $A$. baumannii infection in SOT recipients was $3.9 \%$ (71 of 1,821 ), with rates of $3.1 \%$ in kidney recipients and $8.4 \%$ in liver or simultaneous liver-kidney recipients, in our present study. Although in more transplant centers, including ours, higher-immunosuppression regimens are used in kidneytransplant recipients, the incidence rate of $A$. baumannii 
Table 3 Risk factors for infection-related mortality in SOT recipients with Acinetobacter baumannii infection

\begin{tabular}{|c|c|c|c|c|}
\hline Characteristics & Related mortality & Survival & $P$-value & OR $(95 \% \mathrm{Cl})$ \\
\hline Total, n (\%) & $29(40.8)$ & $42(59.2)$ & & \\
\hline \multicolumn{5}{|l|}{ Univariate analysis, n (\%) } \\
\hline Age $\geq 40$ years & $20(69.0)$ & $29(69.0)$ & 0.994 & $0.99(0.36-2.77)$ \\
\hline Male sex & $21(72.4)$ & $32(76.2)$ & 0.363 & $0.61(0.21-1.78)$ \\
\hline Temperature $\geq 39^{\circ} \mathrm{C}$ & II (37.9) & $6(14.3)$ & 0.026 & $3.67(1.17-11.52)$ \\
\hline Inappropriate antibiotics & $15(5 \mid .7)$ & $26(6 \mid .9)$ & 0.394 & $0.66(0.25-1.72)$ \\
\hline Nosocomial infection & $28(96.6)$ & $36(87.9)$ & 0.165 & $4.67(0.53-41.03)$ \\
\hline Liver or liver-kidney transplant & $10(34.5)$ & $13(3 \mid .0)$ & 0.755 & $1.17(0.43-3.21)$ \\
\hline Multiple culture-positive sites & $9(31.0)$ & $7(16.7)$ & 0.160 & $2.25(0.73-6.97)$ \\
\hline Late-onset infection & $17(58.6)$ & $13(3 \mid .0)$ & 0.022 & $3.16(1.18-8.48)$ \\
\hline Acute rejection & $6(20.7)$ & $7(16.7)$ & 0.667 & $1.30(0.39-4.38)$ \\
\hline Induction therapy & $8(27.6)$ & II (26.2) & 0.896 & $1.07(0.37-3.12)$ \\
\hline Reoperation & $3(10.3)$ & $4(9.5)$ & 0.909 & $1.10(0.23-5.31)$ \\
\hline MDR A. baumannii & $24(82.8)$ & $29(69.0)$ & 0.197 & $2.15(0.67-6.90)$ \\
\hline ICU stay & $28(96.6)$ & $23(54.8)$ & 0.003 & $23.13(2.88-186.10)$ \\
\hline Mechanical ventilation & $26(89.7)$ & $7(16.7)$ & $<0.001$ & $43.33(10.22-183.73)$ \\
\hline Septic shock & 14 (48.3) & $3(7.1)$ & $<0.001$ & $12.13(3.05-48.32)$ \\
\hline Platelet count $<50,000 / \mathrm{mm}^{3}$ & $16(55.2)$ & $8(19.0)$ & 0.002 & $5.23(1.81-15.14)$ \\
\hline Lymphocyte count $<300 / \mathrm{mm}^{3}$ & $13(44.8)$ & $10(23.8)$ & 0.066 & $2.60(0.94-7.21)$ \\
\hline Albumin $<30 \mathrm{~g} / \mathrm{L}$ & $8(27.6)$ & $4(9.5)$ & 0.055 & $3.62(0.97-13.46)$ \\
\hline WBC count $>15,000 / \mathrm{mm}^{3}$ & $9(31.0)$ & $13(3 \mid .0)$ & 0.994 & $1.00(0.36-2.79)$ \\
\hline Creatinine $>1.5 \mathrm{mg} / \mathrm{dL}$ & $21(72.4)$ & $20(47.6)$ & 0.041 & $2.89(1.05-7.97)$ \\
\hline \multicolumn{5}{|l|}{ Multivariate analysis } \\
\hline Mechanical ventilation & & & $<0.001$ & $\begin{array}{l}\mid 89.98(\mid 3.23- \\
2,728.8 \mid)\end{array}$ \\
\hline Platelet count $<50,000 / \mathrm{mm}^{3}$ & & & 0.030 & $12.76(|.28-126.8|)$ \\
\hline
\end{tabular}

Abbreviations: SOT, solid-organ transplantation; OR, odds ratio; $\mathrm{Cl}$, confidence interval; MDR, multidrug-resistant; ICU, intensive care unit; WBC, white blood cell.

infection in liver or simultaneous liver-kidney recipients was higher than that in kidney recipients, because patients undergoing liver or simultaneous liver-kidney transplantation had several conditions that favor postoperative $\mathrm{A}$. baumannii infection, such as preoperative malnutrition due to end-stage liver disease, insulin resistance, major surgical trauma, massive intraoperative bleeding and transfusions, the placement of various catheters, and long duration of antibiotic use before and after transplantation, which could enhance the likelihood of emergence of $A$. baumannii.

Ninety percent of recipients had A. baumannii infection with nosocomial origin in our present study, in alignment with previous studies ${ }^{5,15}$ reporting $A$. baumannii to be almost exclusively a nosocomial pathogen. We found that $74.6 \%$ of recipients developed MDR A. baumannii infection, and the incidence rate of MDR A. baumannii was higher than that of MDR $A$. baumannii reported by Kusne et al (64\%). ${ }^{4}$ The possible reasons to explain this included MDR being defined as resistance to three or more major classes of antibiotics historically effective against $A$. baumannii (fluoroquinolones, carbapenems, aminoglycosides, penicillins, and cephalosporins) in the study conducted by Kusne et al whereas in our present study, MDR was defined as acquired nonsusceptibility to at least one agent in three or more antimicrobial categories, which included nine classes of antibiotics.

Our present study showed that $A$. baumannii infection was associated with high morbidity $(3.9 \%)$, as well as high overall in-hospital mortality (53.5\%) and infection-related 30 -day mortality (40.8\%), in line with previous studies that reported an incidence rate of up to $6.1 \%$ and an extremely high mortality rate of up to $80 \%$ among SOT recipients with A. baumannii infection. ${ }^{5-10}$

Mechanical ventilation associated with both overall inhospital mortality and infection-related 30 -day mortality were in agreement with a recent study claiming that the risk of A. baumannii infection-associated mortality was higher in SOT patients on mechanical ventilation. ${ }^{7}$ Mechanical ventilation support is associated with both overall in-hospital mortality and infection-related 30-day mortality because it probably surrogates markers of clinical severity. ${ }^{7} \mathrm{We}$ also found that late-onset A. baumannii infection was an associated risk factor with overall in-hospital mortality. The association partly reflects the effect of clinical severity on outcomes, given SOT recipients with lateonset $A$. baumannii infection needed more frequent ICU stay ( $80 \%$ vs $66 \%$ ) and ventilator dependence ( $70 \%$ vs $24 \%$ ) when compared with recipients with early onset infection. 
We revealed that liver or liver-kidney transplantation was significantly related to overall mortality, in accordance with Moreno et al who found liver transplantation to be a risk factor for higher mortality in transplant recipients with bloodstream infections. ${ }^{27} \mathrm{We}$ also revealed that thrombocytopenia was a risk factor associated with infection-related mortality. This finding probably reflects a confounding factor, as thrombocytopenia developed more frequently among liver recipients who presented with higher baseline clinical severity. Our findings consolidated results from previous studies of SOT recipients, which reported that lower platelet count to be associated with infection-related mortality. ${ }^{24,28,29}$

To the best of our knowledge, this is the largest study specifically focusing on A. baumannii infections to investigate the epidemiology, distribution, and risk factors for overall in-hospital and infection-related 30-day mortality in SOT recipients. However, the present study has certain limitations. Firstly, our study was a small retrospective chart review with potential limitations, such as data limited to existing medical records. Secondly, for the relatively small number of cases and deaths, the statistical power may be insufficient. Thirdly, we may have underestimated the true rates of $A$. baumannii infections in SOT recipients, given that 1) cases of $A$. baumannii infections outside our institution were not identified, and 2) some cases received empiric courses of antimicrobial therapy before specimens for bacterial culture were obtained. Finally, though we tried to include all relevant data, other unidentified variables are probably risk factors for mortality. The strength of our study is the long study period covering two centers in two different cities in the PRC. However, given the retrospective data collection and risk for bias, all reported findings should be interpreted with care.

\section{Conclusion and recommendation}

High morbidity and high unfavorable outcomes in SOT recipients with infections due to $A$. baumannii were observed. This study confirmed that the risk factors significantly associated with increased mortality in SOT recipients with A. baumannii infection were mechanical ventilation, liver or liver-kidney transplantation, thrombocytopenia, and lateonset infection. Therefore, the clinical and laboratory factors noted in the present study could be useful for clinicians to identify SOT recipients with $A$. baumannii infection who are at high risk for mortality.

\section{Disclosure}

The authors report no conflicts of interest in this work.

\section{References}

1. Dummer JS, Singh N. Infections in solid organ transplant recipients. In: Mandell GL, Bennett JE, Dolin R. Mandell, Douglas, and Bennett's Principles and Practice of Infectious Diseases. 7th ed. Philadelphia: Churchill Livingstone; 2010:3839-3850.

2. Fishman JA. Infection in solid-organ transplant recipients. $N$ Engl J Med. 2007;357:2601-2614.

3. Snyder JJ, Israni AK, Peng Y, Zhang L, Simon TA, Kasiske BL. Rates of first infection following kidney transplant in the United States. Kidney Int. 2009;75:317-326

4. Kusne S, Dummer JS, Singh N, et al. Infections after liver transplantation. An analysis of 101 consecutive cases. Medicine (Baltimore). 1988; 67:132-143.

5. Reddy P, Zembower TR, Ison MG, Baker TA, Stosor V. Carbapenemresistant Acinetobacter baumannii infections after organ transplantation. Transpl Infect Dis. 2010;12:87-93.

6. Shields RK, Clancy CJ, Gillis LM, et al. Epidemiology, clinical characteristics and outcomes of extensively drug resistant Acinetobacter baumannii infections among solid organ transplant recipients. PLoS One. 2012;7:e52349.

7. de Gouvêa EF, Martins IS, Halpern M, et al. The influence of carbapenem resistance on mortality in solid organ transplant recipients with Acinetobacter baumannii infection. BMC Infect Dis. 2012; 12:351.

8. Kim YJ, Yoon JH, Kim SI, et al. High mortality associated with Acinetobacter species infection in liver transplant patients. Transplant Proc. 2011;43:2397-2399.

9. Nunley DR, Bauldoff GS, Mangino JE, Pope-Harman AL. Mortality associated with Acinetobacter baumannii infections experienced by lung transplant recipients. Lung. 2010;188:381-385.

10. Trottier V, Namias N, Pust DG, et al. Outcomes of Acinetobacter baumannii infection in critically ill surgical patients. Surg Infect (Larchmt). 2007;8:437-443.

11. Cisneros JM, Rodríguez-Baño J. Nosocomial bacteremia due to Acinetobacter baumannii: epidemiology, clinical features and treatment. Clin Microbiol Infect. 2002;8:687-693.

12. Linares L, Cervera C, Cofán F, et al. Epidemiology and outcomes of multiple antibiotic-resistant bacterial infection in renal transplantation. Transplant Proc. 2007;39:2222-2224.

13. Falagas ME, Karveli EA. The changing global epidemiology of Acinetobacter baumannii infections: a development with major public health implications. Clin Microbiol Infect. 2007;13:117-119.

14. Morgan DJ, Liang SY, Smith CL, et al. Frequent multidrug-resistant Acinetobacter baumannii contamination of gloves, gowns, and hands of healthcare workers. Infect Control Hosp Epidemiol. 2010;31: 716-721.

15. Abbo A, Navon-Venezia S, Hammer-Muntz O, Krichali T, Siegman-Igra Y, Carmeli Y. Multidrug-resistant Acinetobacter baumannii. Emerg Infect Dis. 2005;11:22-29.

16. Monterrubio-Villar J, González-Velasco C, Valdezate-Ramos S, Córdoba-López A, Villalón-Panzano P, Saéz-Nieto JA. Outbreak of multiresistant Acinetobacter baumannii in a polyvalent intensive care unit: clinical, epidemiological analysis and PFGE-printing evolution. Eur J Clin Microbiol Infect Dis. 2009;28:1281-1284.

17. Munoz-Price LS, Zembower T, Penugonda S, et al. Clinical outcomes of carbapenem-resistant Acinetobacter baumannii bloodstream infections: study of a 2-state monoclonal outbreak. Infect Control Hosp Epidemiol. 2010;31:1057-1062.

18. Kitazono H, Rog D, Grim SA, Clark NM, Reid GE. Acinetobacter baumannii infection in solid organ transplant recipients. Clin Transplant. 2015;29:227-232

19. Horan TC, Andrus M, Dudeck MA. CDC/NHSN surveillance definition of health care-associated infection and criteria for specific types of infections in the acute care setting. Am J Infect Control. 2008;36: 309-332.

20. Gao F, Ye QF, Wan QQ, Liu S, Zhou J. The distribution and resistance of pathogens among liver transplant recipients with Acinetobacter baumannii infections. Ther Clin Risk Manag. 2015;11:501-505. 
21. Lee SO, Kang SH, Abdel-Massih RC, Brown RA, Razonable RR. Spectrum of early-onset and late-onset bacteremia after liver transplantation: implications for management. Liver Transpl. 2011;17:733-741.

22. Wan Q, Ye Q, Yuan H. Multidrug-resistant Gram-negative bacteria in solid organ transplant recipients with bacteremias. Eur J Clin Microbiol Infect Dis. 2015;34:431-437.

23. Kollef MH, Micek ST. Strategies to prevent antimicrobial resistance in the intensive care unit. Crit Care Med. 2005;33:1845-1853.

24. Wan Q, Ye Q, Zhou J. Mortality predictors of bloodstream infections in solid-organ transplant recipients. Exp Clin Transplant. 2013;11: 211-214.

25. Kutinova A, Woodward RS, Ricci JF, Brennan DC. The incidence and cost of sepsis and pneumonia before and after renal transplantation in the United States. Am J Transplant. 2006;6:129-139.
26. van Delden C, Blumberg EA. Multidrug resistant Gram-negative bacteria in solid organ transplant recipients. Am J Transplant. 2009;9 Suppl 4: S27-S34.

27. Moreno A, Cervera C, Gavaldá J, et al. Bloodstream infections among transplant recipients: results of a nationwide surveillance in Spain. Am J Transplant. 2007;7:2579-2586.

28. Nieto-Rodriguez JA, Kusne S, Mañez R, et al. Factors associated with the development of candidemia and candidemia-related death among liver transplant recipients. Ann Surg. 1996;223:70-76.

29. Wan Q, Xiao X, Ye Q, Zhou J. [Risk factors for bloodstream infections in liver or kidney transplantation recipients]. Zhong Nan Da Xue Xue Bao Yi Xue Ban. 2012;37:924-927. Chinese.

\section{Publish your work in this journal}

Therapeutics and Clinical Risk Management is an international, peerreviewed journal of clinical therapeutics and risk management, focusing on concise rapid reporting of clinical studies in all therapeutic areas outcomes, safety, and programs for the effective, safe, and sustained use of medicines. This journal is indexed on PubMed Central, CAS,
EMBase, Scopus and the Elsevier Bibliographic databases. The manuscript management system is completely online and includes a very quick and fair peer-review system, which is all easy to use. Visit http://www.dovepress.com/testimonials.php to read real quotes from published authors.

Submit your manuscript here: http://www.dovepress.com/therapeutics-and-clinical-risk-management-journal 